VII. CONVECTION AND MAGNETIC FIELDS

IN SOLAR-TYPE STARS 


\title{
OBSERVING, MODELING, AND UNDERSTANDING STELLAR GRANULATION
}

\author{
DAINIS DRAVINS \\ Lund Observatory \\ Box 43 \\ $S-22100$ Lund \\ Sweden
}

\begin{abstract}
Numerical simulations of the three-dimensional structure and time evolution of stellar surface convection are now possible, at least for solar-type stars. Using the output from such simulations as sets of spatially and temporally varying model atmospheres, synthetic granulation images and spectral line profiles are computed, and compared to observations. Thus obtained disk-integrated data agree with observed lineshapes and bisector patterns in different stars, and also permit stellar rotation to be determined. Such simulations represent the first generation of models that are free from the classical ad hoc parameters of 'mixing-length', 'micro-' or 'macro-turbulence', parameters which in the past have characterized and limited stellar astrophysics.

To infer the surface structure also in more exotic stars, simpler and parametrized models must still be used to interpret observed line asymmetries. Such models suggest that rapidly rising 'granules' cover only a small surface fraction on early-type stars, a situation opposite to that in solar-type ones, and one likely to affect magnetic fields and stellar activity. Theoretical challenges for the future include detailed modeling also of early-type, giant, and other non-solar type stars of different rotational velocities; the hydrodynamics of entire stellar envelopes (including the interaction with global oscillations); and the interaction with magnetic fields (including their generation). Greatly increased computing power will be needed for such detailed modeling throughout the Herzsprung-Russell diagram, possibly requiring custom-designed computers.

Signatures of stellar granulation are primarily observed as asymmetries and wavelength shifts in photospheric absorption lines. Observational challenges include achieving sufficient spectral resolution to fully resolve such asymmetries; identifying granulation signatures throughout the HR-diagram (including the blended spectra of cool stars); observing how line asymmetries for a given spectral type depend on stellar rotational velocity; measuring wavelength shifts between groups of different lines in the same star, and between different stars; monitoring lineshift variations during stellar activity cycles; and ultimately high-resolution spectroscopy of spatially resolved granulation structures across stellar disks. The latter will require active optics on future very large telescopes, or the use of long-baseline optical interferometers.
\end{abstract}

\section{Introduction}

Stellar granulation has now joined chromospheres and coronae as another 'gift' from solar to stellar astrophysics. Building upon the solar experience, observable effects of stellar surface structure have been identified in stellar spectra, and a first generation of inhomogeneous stellar models has been developed, initiated from their solar counterparts. The aim of this paper is in particular to discuss the future potential of such studies, and to identify directions in which future work should be headed. 


\section{Theoretical Models of Stellar Granulation}

The aims of stellar granulation modeling may be somewhat different from the solar equivalent because of the more fundamental questions that may be posed regarding the surface structure and emission of radiation in widely different types of stars. After all, surface inhomogeneities on the Sun have been studied in great detail for a long time, and with considerable spatial resolution, while the usual description of stellar atmospheres in the past has been by homogeneous models only. Consequently, already rather primitive models might bring interesting new information. On the other hand, the more limited observational data that can be obtained for stars, seriously limits the number of ways in which models can be tested, and makes it practically impossible to obtain unique 'inversions' of observational data into a numerical description of spatially unresolved structures. The degrees of freedom in parametrized models of threedimensional stellar atmospheres are potentially very many, thus preventing the application of traditional modeling techniques (i.e. the adjustment of successive parameters until the output fits the observed data).

Such limitations can (and should) be avoided by instead using computational experiments to numerically simulate stellar surface convection. Although the solar (and, by implication, stellar) surface structure at first sight may appear to be quite complex, the number of fundamental physical processes that determine the structure at large, is probably very limited (at least in non-magnetic regions). This makes the problem well suited to numerical simulations: the finite number of physical processes can be described by laws which are mathematically known and physically understood (e.g. those of hydrodynamics and radiative transfer). The availability of supercomputers then makes it possible to perform numerical simulations with considerable detail and realism. In particular, it becomes possible to model nonlinear and complexly intercoupled phenomena, whose correct interpretation from observations and parametrized models only could be next to impossible. For a general introduction to this new role of supercomputer numerical laboratories, see e.g. Winkler et al. (1987).

Using the output from such simulations as sets of time- and space-dependent model atmospheres, line profiles may be obtained as spatial and temporal averages, allowing a direct test against observations. This confrontation between theory and observation is a most important and crucial step in the development of realistic models, and for the ultimate understanding of stellar atmospheres. During past decades, the development of realistic theories for stellar convection and motions in stellar atmospheres has been severely hampered due to the lack of any sensitive observational verification. Primary among observable parameters are the disk-averaged line profiles, together with their asymmetries and wavelength shifts, and thus theoretical models should be able to confront observations by predicting (at least) these parameters.

\subsection{REALISTIC MODELS OF STELLAR GRANULATION}

While important insights about the processes in stellar photospheres certainly can be gained already through e.g. parametrized, few-component, two-dimensional and/or stationary models, a fully realistic description ultimately demands a time-variable modeling in three dimensions (corresponding to the real world). In recent years it has become possible to solve numerically the sets of hydrodynamic equations that describe the three-dimensional time evolution of solar granular convection (Nordlund, 1982; 1985). The equations of motion (which describe the time evolution of the velocity field) and the energy equation (which describes the time evolution of the temperature field) are used to step a numerical representation of the velocity and temperature fields 
forward in time. The use of realistic background physics (equation of state, absorption coefficients, etc.) taken from standard stellar atmosphere code, a detailed treatment of the radiative transfer (non-grey, three-dimensional), and the use of relevant boundary conditions (in particular the absence of any walls or edges that could artificially constrain the flows), leads to a realistic simulation of the granular convective motions.

Such models naturally contain a number of physical, mathematical and numerical approximations. However, they contain no arbitrary or adjustable physical parameters: the results depend in principle only on the effective temperature, surface gravity and the chemical abundance. (These determine the heat flux into the simulation volume, the gravity forces stratifying the atmosphere, and the opacity of the gases, respectively.)

Using an improved version of these computer codes (initially developed for solar modeling), simulations have been made for models of four different stars (Nordlund and Dravins, 1989). The temperature dependence of granulation along the main sequence is explored with models hotter and cooler than the Sun. $A \mathrm{~T}_{\text {eff }}=6600 \mathrm{~K}$ model has parameters close to those of Procyon (F5 IV-V), while a cooler one with $T_{\text {eff }}=5200 \mathrm{~K}$ corresponds to the $\mathrm{K} 1$ dwarf $\alpha$ Centauri $B$. The luminosity dependence is examined by two models at solar temperature $\left(\mathrm{T}_{\text {eff }}=5800 \mathrm{~K}\right)$ : one at one half solar surface gravity corresponds to the slightly evolved main-sequence star $\alpha$ Centauri $A$ (G2 V), while another at one quarter solar surface gravity has parameters similar to the subgiant $\beta$ Hydri (G2 IV). The simulations were made with a $32 \times 32 \times 32$ spatial grid: $32 \times 32$ Fourier components horizontally, and 32 knot points defining cubic splines for the vertical dependence of different parameters.

This small grid of models thus allows a first study of the temperature and luminosity dependence of granulation in the vicinity of the Sun in the Hertzsprung-Russell diagram. Using the output from these simulations as sets of spatially and temporally varying model atmospheres, synthetic stellar surface images and spectral line profiles were computed for a series of different parameters. This permits a study of continuum and spectral line formation in inhomogeneous stellar atmospheres, and also allows a comparison with observed line profiles and asymmetries (Dravins and Nordlund, 1989a; 1989b). Samples from this work are used to illustrate the discussion below.

\subsection{THE FORMATION AND EVOLUTION OF STELLAR GRANULATION}

In the surface regions of all models, hotter and generally rising features are seen, analogous to solar granules. The granulation phenomenon turns out to be closely connected to the temperature sensitivity of the stellar opacity (in particular that of the negative hydrogen ion, $\mathrm{H}^{-}$), and less dependent on e.g. the stellar surface temperature. When the temperature in the hot and rising elements falls below $\simeq 10,000 \mathrm{~K}$, the opacity is rapidly reduced, the radiation detaches from the gas, and the temperature quickly drops to $\approx 6000 \mathrm{~K}$. The granulation patterns are most pronounced in these regions where radiation detaches from matter. In the hotter Procyon model $\left(\mathrm{T}_{\text {eff }}=6600 \mathrm{~K}\right)$, this region includes the visible stellar surface, which is thus covered by 'naked' granules of very high temperature contrast. In solar-temperature stars, this region is (barely) beneath the surface. Although the granulation pattern is still visible on the surface, the temperature contrast increases with depth. In the cooler $\left(T_{\text {eff }}=5200 \mathrm{~K}\right)$ star, however, this sharp temperature drop remains 'hidden' beneath the visible surface, to which only low-contrast temperature features are able to penetrate. In this case, the radiation is unable to directly escape, but is rather largely absorbed in the upper photosphere, whose temperature structure is strongly influenced by this absorption of radiation from below.

The normal evolution of stellar granules is characterized by a gradual increase in size until they disintegrate due to various causes. Relatively undisturbed granules often grow 
until they 'collapse under their own weight'. In order to carry the stellar convective heat flux outward, there must be an upflow of hot gases at a few $\mathrm{km} / \mathrm{s}$ from beneath the photosphere. Upon reaching optically thin layers, these gases cool off and eventually turn around to descend in the cooler intergranular areas. The necessary horizontal acceleration of the gases toward the sinking areas is accomplished by local overpressures that develop over granules (and also over intergranular lanes, in order to decelerate the incoming horizontal flow there). The larger the granule, the larger the overpressure required to accelerate away the material over the greater horizontal distances. Ultimately, the overpressure on the surface becomes so great that it impedes the arrival of additional hot gases from below. Having been cut off from its energy supply, the granular center begins to cool off, a dark center develops, and the now ring-shaped granule disintegrates, a process apparently similar to solar exploding granules. This mechanism limits the characteristic scale of granules on different stars: the granules cannot grow any larger because the required overpressure would block further convective energy supplies from beneath, thus strangling the granule through a lack of input heat. Characteristic scales range between $\approx 10^{3} \mathrm{~km}$ in the cool $\alpha$ Cen $B$ model to $\approx 10^{4} \mathrm{~km}$ on the subgiant $\beta$ Hyi.

Not all granules evolve in this manner. Some granules that are disturbed by velocity flows in neighboring granules may cleave and break apart into smaller fragments, which in turn grow and merge into new features. Some periods may be characterized by especially vigorous granulation with large and well correlated temperature and velocity amplitudes, while other periods (especially following the breakup of some particularly large granule) may be characterized as 'abnormal', with apparently chaotic temperature and velocity fields, as the photosphere is gradually returning back to a more stable situation (Nordlund and Dravins, 1989).

\subsection{THE SURFACE STRUCTURE OF VELOCITY AND TEMPERATURE}

As an example of stellar surface structure, Fig. 1 shows a 'snapshot' of the Procyon model at a representative instant, i.e. at a time when its spatially averaged properties are roughly similar to the average for the entire simulation sequence. Temperature, pressure and velocities are shown at constant geometrical depth at the stellar 'surface'. (This is here defined as the deepest horizontal level in the simulation volume where the radiative flux exceeds $50 \%$ of the total energy flux.) The temperature structures are sharply delineated and correlate well with rising velocities. The pressure patterns are smoother and display the overpressures discussed above. The horizontal velocity field is obviously directed away from the upflows into the downflow regions. The occasional lack of a detailed correlation with the superposed 'granular' contours is due to the time history of the different features: old and decaying granules may already begin to be swept away by horizontal flows originating elsewhere. The vertical cuts into the simulation volume (bottom panels) show in particular the asymmetries in the vertical flows. While the upflows are relatively gentle and spread out over extended volumes, the sinking material rapidly converges into concentrated and strong downflows that preserve their identity to large depths. Gases in these downflows often develop a significant rotational motion beneath the surface (by conserving angular momentum from random surface motions), reminiscent of terrestrial tornados. Analogous to the solar case (Nordlund, 1985), a large fraction of the convective energy flux is carried in these cool and concentrated downflows which occupy only a small fraction of the surface area: stellar convection is thus a highly inhomogeneous and intermittent process.

Finally, it can be noted in the vertical cuts in Fig. 1 that the stellar 'surface' (now defined by the $\mathrm{T}_{\text {eff }}=6600 \mathrm{~K}$ level) is highly 'corrugated' with an amplitude of perhaps 

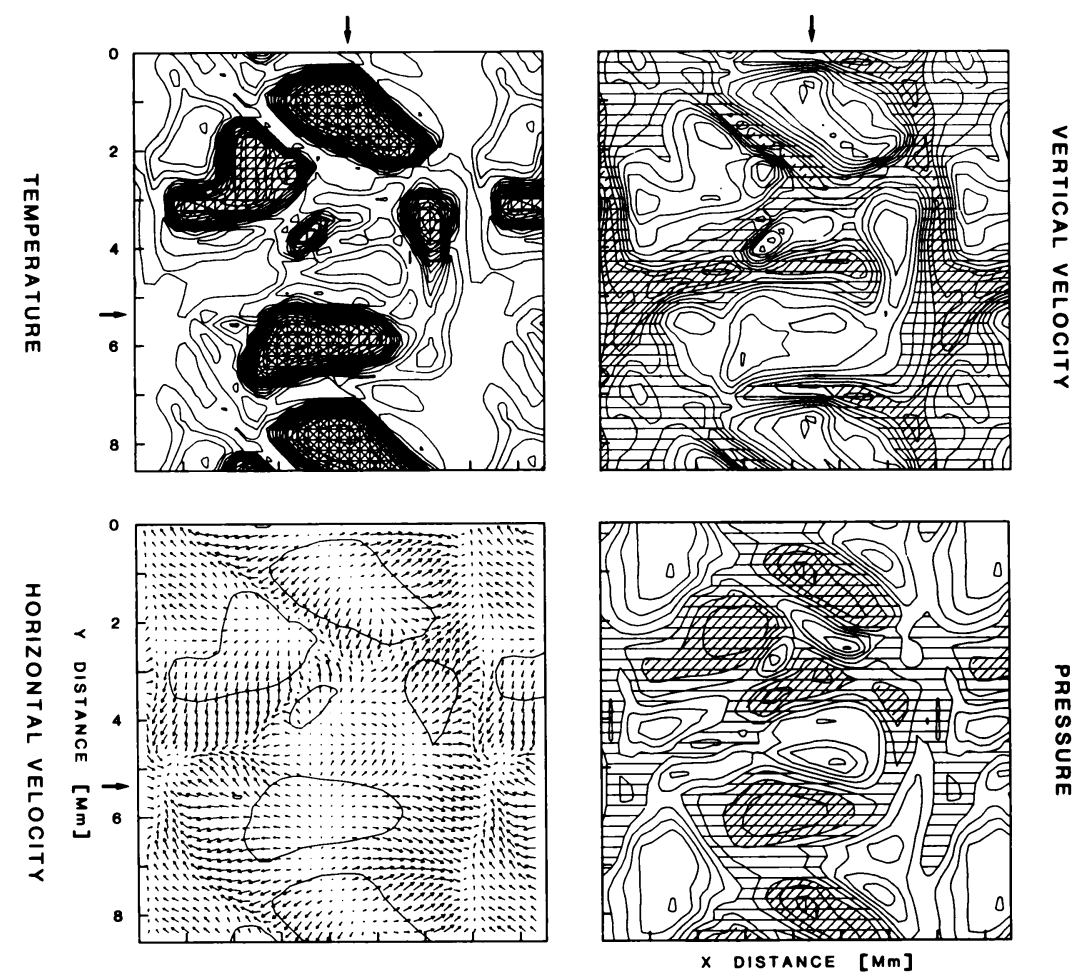

0
0
0
0
0
0
0
0
$m$
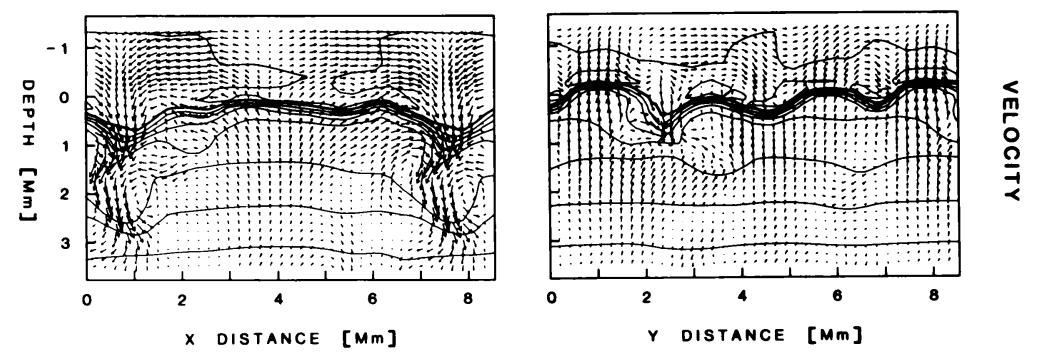

Figure 1. Overall appearance of the Procyon simulation at a representative time. The top four panels show temperature, pressure and velocities at a fixed geometrical height at the stellar 'surface'. Temperatures are shaded above the $T_{\text {eff }}$ value of $6600 \mathrm{~K}$, with contours every $500 \mathrm{~K}$ and shading increasing every $1500 \mathrm{~K}$. Downward velocities are shaded with contours every $\mathrm{km} / \mathrm{s}$ and shading in steps of $3 \mathrm{~km} / \mathrm{s}$. Horizontal velocity vectors correspond to 40 seconds of gas motion. The superposed $T_{\text {eff }}=6600 \mathrm{~K}$ contours delineate the granules. The logarithmic pressure plot is shaded above $10^{3.6} \mathrm{~Pa}$, has contours every $0.05 \mathrm{dex}$, and increased shading every $0.1 \mathrm{dex}$. The Fourier representation makes the images periodic in $X$ and $Y$, with a cycle of $\simeq 6840 \mathrm{~km}$ (slightly more than $5 / 4$ of one cycle is shown). The two bottom panels show the velocity components in vertical planes. The left cut into the simulation volume (at $Y \simeq 5.3 \mathrm{Mm}$, as marked by arrows) goes across a large growing granule and its associated downflows, while the right one $(X \approx 4.3 \mathrm{Mm})$ goes across different smaller granules. The bold temperature contour marks $\mathrm{T}_{\text {eff }}=6600 \mathrm{~K}$, with other contours at $1500 \mathrm{~K}$ intervals. (Nordlund and Dravins, 1989) 
$500 \mathrm{~km}$. This is a particular feature of this hotter model (rather less pronounced in the cooler ones), and leads to several consequences in the optical appearance of granulation across the stellar disk, as well as in asymmetries and shifts of the line profiles. Such a 'corrugated' surface on F-stars could be predicted already in a simpler granulation model by Nelson (1980). Another model for granulation on Procyon was presented by Atroshchenko et al. (1989).

\section{The Appearance of Stellar Surfaces}

The output data from the numerical simulations were used as input for radiative transfer calculations to obtain the emerging continuum and line radiation for different spatial points, angles, wavelengths, and spectral line parameters. In Fig. 2, synthetic images are shown for the Procyon model, at the same representative instant of time as in Fig. 1. As expected, the geometrical shapes of granules at stellar disk center closely correspond to the temperature features. However, the intensity contrast is much lower than could have been naively expected from the temperature contrast at a constant geometrical depth: the temperature-dependent opacities of overlying layers hide the larger temperature contrasts beneath. Nevertheless, by solar standards, the intensity contrast is rather high, and furthermore increases toward the stellar limb. This increase originates from the
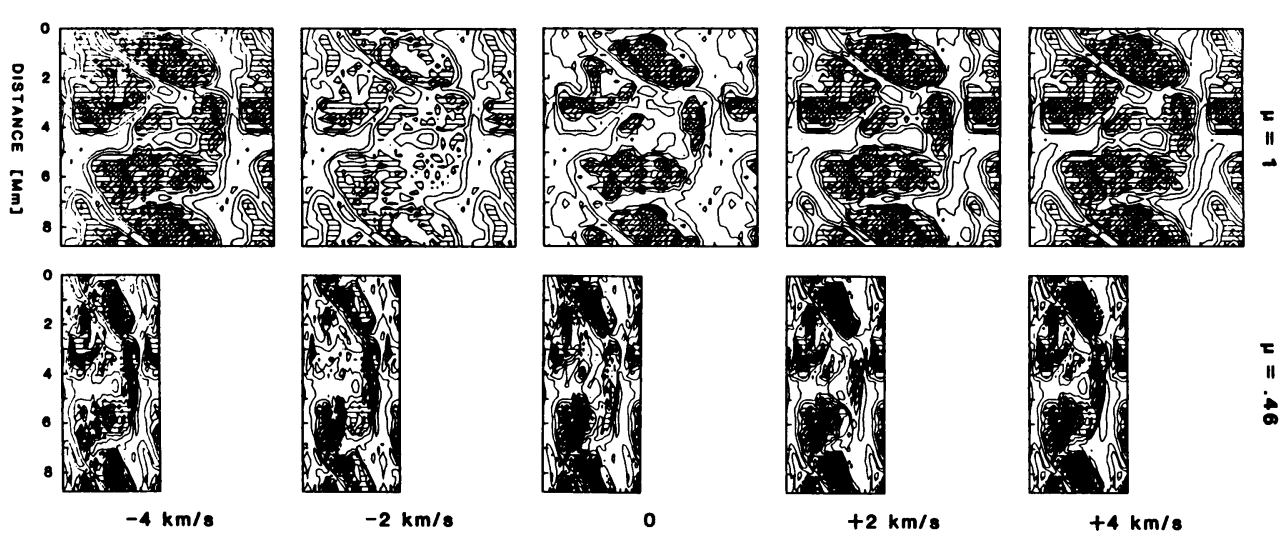

Figure 2. Synthetic monochromatic images of Procyon at the same time as Fig. 1. The monochromatic brightness across a weak Fe I line of $\chi=3 \mathrm{eV}$ at $\lambda 520 \mathrm{~nm}$ is converted to images seen through an ideal narrow-band monochromatic filter, scanning across the line. The region is shown both at disk center $(\mu=\cos \theta=1)$ and as seen (from the left) toward the limb $(\mu=0.46)$. Areas brighter than average are shaded, with contours at every $20 \%$ of the average. Noteworthy is the high granulation contrast, which increases from disk center toward the limb. This reflects the 'corrugated' surface in this $T_{\text {eff }}=6600 \mathrm{~K}$ model which, under large inclination angles, occasionally makes it possible to view hot bright spots in deeper layers. As on the Sun, the granulation contrast at disk center has a maximum in the red wing of the line, while the changes with wavelength are much smaller near the limb. This reflects the weak brightness-velocity correlation for the horizontal velocities seen close to the limb. A further discussion is in Dravins and Nordlund (1989a). 
'corrugated' nature of the Procyon surface which, at larger inclination angles, occasionally makes it possible to glimpse hot and bright elements in deeper layers. This particular property is not shared by the cooler models, and the granulation contrast in e.g. the $\mathrm{T}_{\text {eff }}=5200 \mathrm{~K}$ model is considerably lower and shows no tendency to increase toward the limb.

\subsection{LINE FORMATION IN STELLAR PHOTOSPHERES}

The simulation data can in particular be used as an input to compute photospheric line profiles at different spatial locations and at different times. The subsequent analysis of such profiles can give considerable insight in the physics of line formation in inhomogeneous stellar photospheres and can predict observable quantities which can verify (or falsify) different models. Further, it may indicate the validity (if any) of classical approximations such as the concepts of 'micro-' or 'macro-turbulence'.

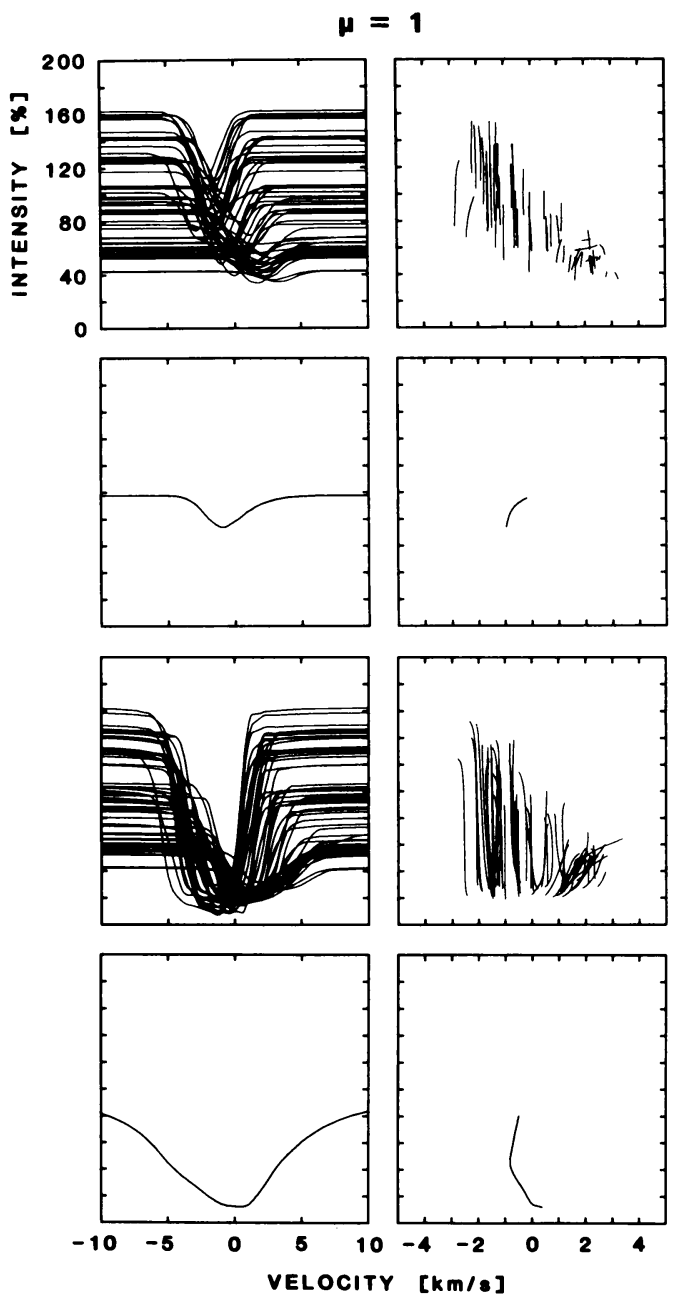

Figure 3. Spatially resolved line profiles at $\alpha$ Cen $A$ disk center, and their spatial averages at a representative time. This model differs from the Sun only through a halved surface gravity. Line profiles and bisectors are shown for a grid of $8 \times 8=64$ spatial points out of the $32 \times 32=1024$ actually computed at each step in time. The top half of the figure shows data for a weak Fe $I$ line, and the bottom for a very strong one, both with $\chi=3 \mathrm{eV}$ at $\lambda 520 \mathrm{~nm} .100 \%$ intensity corresponds to the average for the entire simulation sequence. The spatially averaged profile and its bisector at this instant in time are also shown. The average profile is not representative for spatially resolved points on the star: its width and asymmetry rather reflect the statistical distribution of spatial inhomogeneities. The redshifted profiles of strong lines from intergranular regions are often asymmetric due to vertical velocity gradients over their extended heights of formation. (Dravins and Nordlund, 1989a) 
Figure 3 shows a sample of such spatially resolved line profiles (and their averages) at a representative time at the disk center of the $\alpha$ Cen $A$ model. An important conclusion from all stellar simulations is that the spatially averaged profile is not at all typical for individual points on the stellar surface. The shape, asymmetry and shift of the average profile instead reflect the statistical distribution functions of different profiles from different spatial points. The pronounced asymmetry of the spatially averaged profile is not frequent: it only occurs where there happen to be strong velocity gradients in the line-forming layers. Such intrinsic asymmetries are more common for strong lines with extended heights of formation, in particular in intergranular lanes. There the downflow velocities rapidly increase with depth, and this depth gradient can be manifest as asymmetries also in spatially resolved lines. This means that attempts to deduce stellar photospheric structure by interpreting observed line asymmetries as arising in horizontally homogeneous models, will likely lead to fortuitous depth-dependent velocity fields that are not at all present in any real stellar atmosphere.

The correlation between brightness and lineshift is particularly well visible for weaker lines (top panel in Fig. 3). This of course indicates that hot elements generally are rising, and the correlation decreases near the stellar limb, where one mainly sees the effects of horizontal velocities. Closer to the limb, there is a noticeable increase in the velocity spread of the individual lines, reflecting that the horizontal velocities are generally of somewhat larger amplitude than the vertical ones.

\subsection{LINE PROFILES IN INTEGRATED STARLIGHT}

By a suitable summation of time-averaged data for different center-to-limb positions, disk-integrated line profiles are obtained. These profiles can be compared to observations, and constitute the most important diagnostic tool for stellar granulation studies. For the four models computed, the most pronounced differences in resulting line shapes are between those in different stars. However, for any given star, line asymmetries and wavelength shifts depend systematically on the line's atomic parameters, analogous to the solar case.

The largest differences are seen among lines of different strength, reflecting different average heights of formation. The photospheric structure changes rapidly with height, and already slight differences in line formation conditions may lead to observable differences. For lines of a given strength, potentially observable differences exist among lines of different excitation potentials and/or ionization levels. These differences originate from the temperature sensitivity of the lines: those of a certain excitation potential will predominantly form in regions whose temperatures are sufficient to strongly populate the relevant atomic energy level, yet not so high as to ionize the species. Generally, Fe I lines of higher excitation potential show a larger blueshift, reflecting the more rapidly rising motion of the hotter elements, where these lines predominantly are formed. Smaller differences exist among otherwise similar lines in different wavelength regions. Such differences occur primarily because the granulation contrast changes with wavelength, and also because of changing continuum opacities. It is through such arrays of differential spectral line behavior that also detailed models of stellar granulation structure can be tested against observations.

\subsection{COMPARISON TO OBSERVATIONS}

The highest-resolution data available on stellar photospheric line profiles and their asymmetries have been obtained with the double-pass scanner of the coude echelle 
spectrometer at European Southern Observatory (Dravins, 1987b). The synthetic line profiles for disk-integrated starlight were convoluted with profiles corresponding to the measured instrumental ones, but slightly broadened to include effects of plausible amplitudes of stellar surface oscillations. For each value of the stellar rotational velocity, this produces a grid of line profiles and bisectors for differently strong spectral lines. Observed line profiles and bisectors were then independently compared against these grids, and 'best-fit' values of $V \sin i$ deduced. Figures 4-5 show the resulting comparison between synthetic and observed data for the well-observed solar near-twin $\alpha \operatorname{Cen} A$.

Despite the similarity to the Sun, the strongest lines in $\alpha \operatorname{Cen} A$ are clearly more asymmetric than corresponding solar ones (Fig.5; compare with solar bisectors plotted

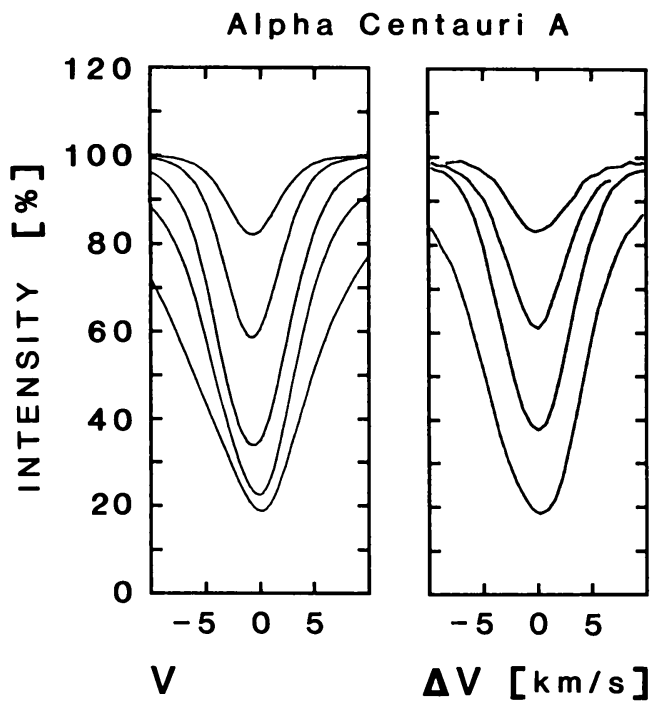

Alpha Contauri A

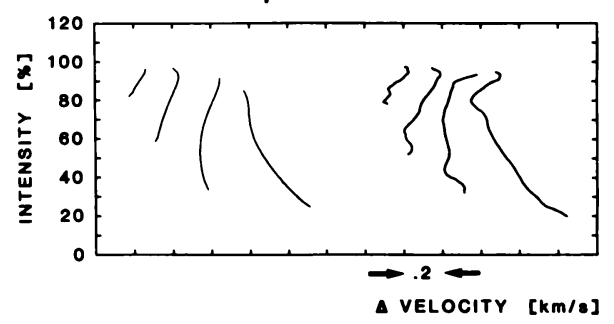

Figure 4. Comparison between 'best-fit' synthetic and observed line profiles for the G2 V star $\alpha$ Cen $A$. Synthetic Fe I lines $(x=3 \mathrm{eV})$ with zero-rotation depths 20 , $45,69,80$ and $83 \%$ of the flux continuum at $\lambda 520 \mathrm{~nm}$ are shown at left, and Fe I line profiles, observed with a resolution $\lambda / \Delta \lambda \simeq 200,000$ are at right $(\lambda 685.57$; $543.63 ; 633.53$; $543.45 \mathrm{~nm}$; for details see Dravins, 1987b). From a grid of rotationally broadened profiles, a 'best-fit' rotation $V \sin i \approx 1.8 \pm .3 \mathrm{~km} / \mathrm{s} \quad$ was obtained. The detailed agreement between observed and synthetic profiles is obtained without involving any classical parameters such as 'mixing-length', 'micro-', nor 'macrouubulence'. (Dravins and Nordlund, 1989b)

Figure 5. Comparison between observed and 'best-fit' synthetic bisectors for $\alpha \operatorname{Cen} A$, analogous to Fig.4. A bisector grid gives a best-fit stellar rotation $V \sin i \approx 1.7 \pm .3$ $\mathrm{km} / \mathrm{s}$, independently of profile fits. The observed bisectors are averages for Fe I lines from Dravins (1987b). The detailed agreement shows that also the asymmetric part of the $\alpha$ Cen $A$ lines is well modeled. This model differs from the solar one only by slightly lower surface gravity. This leads to a slightly more vigorous convective overshoot, causing a more pronounced asymmetry in the cores of the strongest lines; cf. Fig. 3. (Dravins and Nordlund, 1989b) 
in the same format in Dravins, 1987b). This difference to the Sun can be traced back to be an effect of the lower surface gravity in $\alpha \operatorname{Cen} A$, which permits a more vigorous convective overshoot and slightly larger velocity amplitudes in the high photosphere, where the cores of strong lines often are formed. These velocities give contributions to the absorption not only near the spatially averaged line core, but also further out in the line flanks, in a manner to produce this distinct bisector signature (cf. bottom panels in Fig. 3). It is encouraging that current observations and models permit the study of such subtle differences between the velocity fields in two G2 V stars, revealing the effects on solar granulation of some three billion years of stellar evolution.

\section{Understanding Granulation in Different Types of Stars}

Powerful as numerical simulations may be, they still have limitations in modeling granulation in situations very different from the solar one. The problems arise mainly from the extensive computing power required, and the multidimensional nature of the problem. This makes it computationally very expensive to e.g. greatly increase the number of spatial resolution points to include features of all significant (but unknown) geometrical scales. It is not yet practical to produce a grid with a large number of granulation models throughout the Herzsprung-Russell diagram, and many theoretical challenges remain.

\subsection{THE GEOMETRIC SCALE OF GRANULATION}

Numerical simulations confirm that an important factor determining the geometric scale of granulation is the density scale height (at least in solar-type stars). Since that increases greatly from main-sequence stars to giants and supergiants, one may suspect
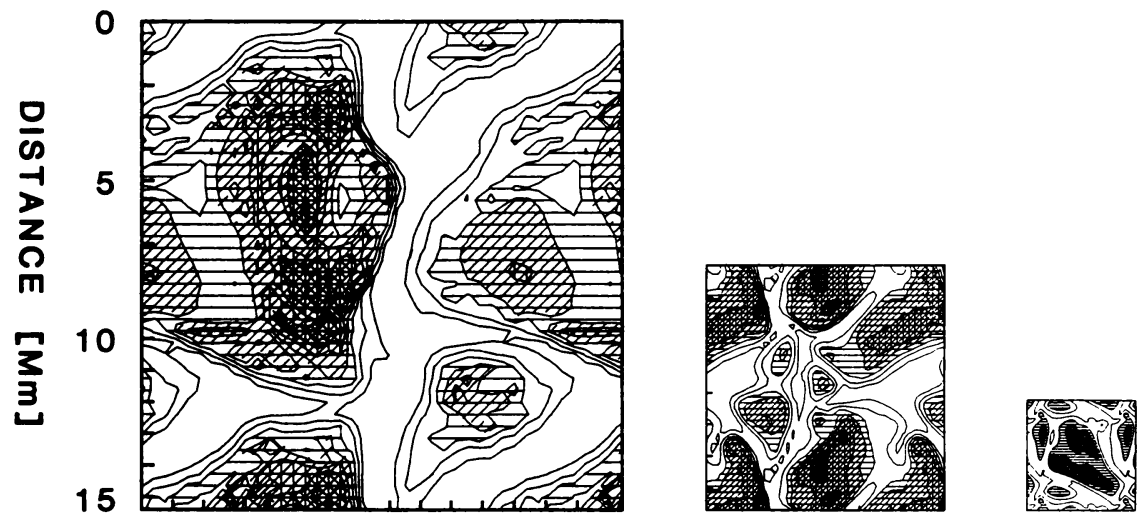

Figure 6. The optical appearance of typical granulation patterns at stellar disk center in different stars, plotted on the same geometric scale. From left to right are models corresponding to $\beta \mathrm{Hyi}$ (G2 IV), $\alpha$ Cen $A$ (G2 V), and $\alpha \operatorname{Cen} B$ (K1 V). Areas brighter than average are shaded, with contours at every $20 \%$ of the average. These synthetic continuum images at $\lambda 520 \mathrm{~nm}$ illustrate the increase in the geometric scale of granulation with increasing density scale height. Adapted from Dravins and Nordlund (1989a). 
that granules on giant stars could be very large, perhaps so large that only a small number of them exist on the entire stellar surface (Antia et al, 1984; Schwarzschild, 1975). Figure 6 shows typical 'snapshots' of granulation images from three stellar models, plotted on a constant geometric scale. Clearly, already in this small region of the Herzsprung-Russell diagram, there is a significant change in granular sizes. One can imagine many different processes affecting granules in giant stars, and their detailed modeling remains a challenge for the future.

\section{2. 'INVERSE' LINE ASYMMETRIES IN EARLY TYPE STARS}

While solar and cool-star bisectors often are similar to the letter ' $c$ ', bisectors in early-type stars often show the inverted ' $J$ ' shape. Indeed, there seems to be a 'granulation boundary' in the Herzsprung-Russell diagram separating these types of bisectors, running from $G$ supergiants to near F0 on the main sequence (Gray and Nagel, 1989). This boundary largely coincides with that of readily observable chromospheric emission (Dravins, 1981). The generally more rapid rotation in early main-sequence stars makes their spectra more difficult to study, but there are indications of similarly inverted asymmetries in the relatively sharp-lined spectra of the dwarfs Sirius (A1 V; Dravins, 1987b) and r Sco (B0 V; Smith and Karp, 1978; 1979).

While one does not expect 'ordinary' surface convection driven by hydrogen ionization in the atmospheres of such hotter stars, there is no reason to believe their atmospheres should be static. Organized motions could well arise due to the $\mathrm{He}$ II ionization zone just below the photosphere, or through some other mechanism. Hydrodynamic models for the A0 V star Vega by Gigas (1989) indeed show its atmosphere to develop an oscillatory behavior, leading to 'inverse' line asymmetries.

An application of simple few-component models, reproducing the forcefully inverted 'inverse' bisector shapes in F supergiants, suggests that their surfaces may be covered by small 'granules', rising rapidly with perhaps $10-20 \mathrm{~km} / \mathrm{s}$ (Gray and Toner, 1986; Dravins, 1989). A visualization of the surfaces of such stars, featuring rapidly upwelling currents in small ascending 'geysers', whose output is balanced by a more sluggish downdraft over more extended areas, is certainly quite different from the familiar image of solar granulation. However, to verify whether such a scenario is correct, requires much more detailed modeling.

\section{Challenges in Stellar Granulation Modeling}

Clearly, detailed granulation modeling should be extended across the HertzsprungRussell diagram, and these simulations should be performed without the simplifying assumptions used up to now. In a shorter time perspective, an important improvement will be to include effects of wave propagation and shock formation. Current models strongly suggest that there is material moving at near-sonic velocities in stellar photospheres, at least in the vigorous granulation on hotter and on subgiant stars. Also, a study of the interaction between stellar granulation and magnetic fields should before long clarify the characteristics of small-scale flux concentrations in different types of stars. In a longer time perspective, one very important challenge can be identified:

\subsection{MODELING ENTIRE STELLAR ENVELOPES}

Current models have only treated very small simulation volumes, corresponding to (at most) a few granules, and not extending very deep into the star. Such simulations clearly 
are unable to model larger-scale structures, such as stellar supergranulation or possible giant cells. While they do show the convective overshoot in the upper photosphere, and its effects on spectral line profiles, the corresponding effects at the bottom of the convective zone (so important for chemical mixing and for understanding stellar evolution) are not modeled. A logical next great step in stellar astrophysics would be the simulation of entire stellar envelopes. Such simulations should predict new types of observable parameters such as the temporal power spectrum of global irradiance fluctuations at different wavelengths, or the power in different $p$-modes of the stellar global oscillations, as well as their typical lifetimes. The capacity of near-future very large ground-based telescopes, as well as planned space instrumentation should permit the accurate observation of such parameters, and thus both demand their modeling, and assure the necessary confrontation between theory and observations.

\subsection{NEW COMPUTATIONAL TECHNIQUES}

There is a need for greatly increased computing power to handle more realistic astrophysical situations, such as the modeling of entire stellar envelopes. Compared to current simulations, the desired increase in stellar area coverage is a factor $\approx 10^{6}$, a factor $\approx 10-100$ in the vertical extent, and $\approx 10$ in the spatial (and possibly also temporal) resolution. The latter is needed in particular to handle wave motion and shock formation. Unless radically more efficient program codes can be invented, there is a need for perhaps $\approx 10^{9}$ more computing power than used at present. At first sight this may appear utterly discouraging since computer performance normally increases by only one order of magnitude from one generation of supercomputers to the next. However, such an ambition level may not be entirely unrealistic.

Workers in computer science are currently putting considerable effort into the development of various 'non-classical' concepts, e.g. employing novel computer architecture with many processors working in parallel. (For a general introduction to such concepts, see e.g. Bowler et al, 1987.) The greatly improved possibilities for computer-aided design and manufacture of unique electronic circuits at not unreasonable cost promises to make it possible to build a computer with circuits custom-designed for solving the problem of stellar convection. The combination of such circuits with novel computer architecture might well improve computing power by several orders of magnitude over today's 'supercomputers'. For example, a dedicated numerical laboratory with 4000 custom-designed CPU's running for three years, will offer more than 100 million CPU-hours, a computing power that may well be adequate to begin realistic simulations of entire stellar atmospheres.

\section{Challenges in Stellar Granulation Observations}

Effects from stellar granulation can be observed through various subtle signatures in high resolution spectra of integrated starlight, through the time variability of stellar irradiance, and by a few other means. The most accessible observational parameter appears to be that of photospheric absorption line asymmetries. Several authors have now reported observations of such asymmetries, and measurements of relative wavelength shifts between different classes of lines are also becoming available (e.g. Dravins, 1982; 1987a; 1987b; Gray, 1988; and references therein). In the future, we can expect additional data from (a) space-based stellar photometry with micromagnitude precision, allowing the observation of time variability of stellar irradiance due to the time evolution of granular features; (b) space-based astrometric measurements of stellar 
radial velocities (not involving the stellar spectrum), thus allowing the determination of absolute convective wavelength shifts; (c) the use of active optics on the very large telescopes now under construction, which should allow diffraction-limited imaging of surface features on giant stars as well as spatially resolved spectroscopy across stellar disks; (d) high-resolution imaging of stellar surfaces that is now becoming feasible through the development of long baseline optical interferometry and optical aperture synthesis.

\subsection{OBSERVING LINE ASYMMETRIES}

The faithful observation of the subtle photospheric line asymmetries caused by stellar granulation is a more demanding task than most other stellar spectroscopy applications. In particular one can point to the issues of:

6.1.1. Finite Spectral Resolution. The asymmetries of spectral lines are often represented by their bisectors. In order to 'fully' resolve the bisector shape, some $4-5$ independent points on the curve may be required. Since the relevant width of most photospheric lines in ordinary stars is on the order of $8-10 \mathrm{~km} / \mathrm{s}$, and each bisector point is obtained from two intensity measurements in opposite flanks of the line, this demands $8-10$ points across the line, corresponding to a resolution of $\simeq 1 \mathrm{~km} / \mathrm{s}(\lambda / \Delta \lambda \simeq 300,000)$. Numerical simulations of how bisector shapes are distorted by a lower resolution clearly illustrate this point (Dravins, 1987a; Livingston and Huang, 1986).

Such resolutions are seldom available in stellar spectrometers, where $\lambda / \Delta \lambda \approx 100,000$ is more common. Although such a resolution may be adequate to detect the presence of line asymmetries, the study of their detailed shapes or the detection of any differences between different types of lines becomes marginal. The fundamental problem is not the lack of spectroscopic instruments of sufficient performance, but the fundamentally poor light efficiency of those that are technically sufficient. For example, Fourier transform spectrometers (FTS) offer fully adequate spectral resolution, a good instrumental profile, possibilities for accurate wavelength determination, and other valuable features. The problem is that an FTS, scanning successive Fourier components of absorption-line spectra in the optical, is photon-noise limited, analogous to a wavelength-scanning single-channel spectrometer. (This is because all the light in the instrument falls onto the detector, and contributes photon noise from all wavelengths all the time, while the desired signal is only a slight modulation on top of this.) In order to use FTS's for stellar work one is forced either to accept a lower spectral resolution or to introduce rather narrow bandpass-limiting wavelength filters. Even so, the observation of only a few spectral lines in very bright stars may require several hours (e.g. Wayte and Ring, 1977; Nadeau and Maillard, 1988).

Fortunately, there is reason for optimism due to the current wave of construction of very large telescopes in different parts of the world. Their greatly increased light collecting power will overcome the photon limits in today's 'large' telescopes, and make Fourier transform spectrometers generally available for stellar spectroscopy. An estimate of the likely performance of an FTS on an 8-10 meter class telescope shows that, at spectral resolving power $\simeq 10^{6}$, a signal-to-noise ratio of 100 should be reached for an $m_{v}=3$ star in one night's integration over a bandpass of $10 \mathrm{~nm}(100 \AA)$. Although some astronomers might still think of $m_{\mathrm{v}}=3$ as representing rather bright objects, the number of stars whose spectra could be studied in great detail will now be counted in the hundreds rather than the handful of the very brightest ones that at present are barely accessible to such studies. 
6.1.2. Problems in Stellar Spectra. Although spectral lines to be studied obviously are selected to be as undisturbed as possible, they are almost never completely unblended. This makes it awkward to draw conclusions on line asymmetries from measurements of very few lines only: how is one to know if an asymmetry is intrinsic to the stellar photosphere or due to a blend? These problems become especially pronounced for measurements close to the continuum: in weak lines or in the wings of stronger ones. Since one can expect blending lines to be randomly positioned in the wings of different primary lines, one solution is to simply average the asymmetries of several similar lines.

If very high spectral resolution is required, this is not a trivial requirement, since each line to be observed might require hours of observing time. In cooler and/or more rapidly rotating stars it may in practice be impossible to find any significant number of sufficiently undisturbed lines. Solutions could possibly include going to the infrared (where line densities are lower), or face the problem of observing (and modeling!) not individual spectral lines, but rather line complexes. For very cool stars this could even be the only alternative. Such complexes of blended lines will carry much the same information as individual lines, but the observational possibilities of averaging bisectors of many similar lines will disappear. Likewise, accurate laboratory wavelengths will be needed for all components making up the blend since average values over similar lines will not suffice.

\subsection{DETERMINING WAVELENGTH SHIFTS}

Since stellar spectral lines are intrinsically asymmetric, their 'wavelengths' can not simply be defined as single quantities. Ideally, line profiles and their bisectors should be measured on an absolute wavelength scale that allows the determination of convective lineshifts in different parts of the line, relative to the line's laboratory wavelength (corrected for the stellar radial velocity and its gravitational redshift). Such accurate wavelengths in spectral lines form important constraints on different theoretical models, which sometimes may even predict the same line asymmetry, but different wavelength shifts. This observational task presents several challenges:

6.2.1. Wavelength Dependence on Spectral Resolution. The finite spectral resolution in stellar spectrometers causes wavelength shifts of stellar lines. The instrumental convolution of an asymmetric stellar line profile with even a perfectly symmetric instrumental one results in a line profile of different asymmetry, where different parts of the bisector now are at different wavelengths. Since e.g. differently strong lines have different intrinsic asymmetries, they will be differently affected by the same spectral resolution, possibly mimicking the astrophysically expected behavior between different groups of lines. For solar-type asymmetries, such instrumentally induced shifts may reach $100 \mathrm{~m} / \mathrm{s}$ for resolutions $\lambda / \Delta \lambda \simeq 100,000$ (Bray and Loughhead, 1978; Dravins, 1987a; Livingston and Huang, 1986).

6.2.2. Wavelength Shifts Between Different Lines in the Same Star. Convective wavelength shifts can be expected to be similar for groups of lines with common properties. Since only statistical data for groups of lines are required, it is not really necessary to record the full stellar spectrum. Rather, it could suffice with data from radial velocity measuring machines, utilizing the cross-correlation of the stellar spectrum with some spectrum template. The well-known instruments of the Griffin- and CORAVEL-type employ hardware masks and reach precisions on the order of $100 \mathrm{~m} / \mathrm{s}$, adequate to begin searches for signatures from stellar granulation.

With different masks, e.g. such preferentially selecting high- or low-excitation lines, 
one could detect differential radial velocities between groups of different lines in the same star. Next-generation radial velocity instruments are likely to avoid mechanical masks in order to improve light efficiency and allow the integration of the full spectrum all the time. Their spectrum templates will then be defined in software by selecting features in the spectrum for cross-correlation. Although there are likely to be many practical hurdles, it should in principle be straightforward to define different templates by selecting interesting groups of lines (from either observed or synthetic; stellar or laboratory spectra) to search for signatures from stellar granulation.

6.2.3. The Need for Very Accurate Laboratory Wavelengths. The most useful atomic species for convective lineshift studies appears to be iron. It has high atomic mass (minimizing the thermal broadening of the stellar lines), its hyperfine and isotope splitting has few complications from atomic and isotope structure, and it has a rich and well-studied spectrum. In the Sun and solar-type stars, there are approximately 500 'unblended' Fe I and 50 Fe II lines in the visual spectrum, with another 100 or so lines in the infrared. Reasonable laboratory wavelengths exist, and convective lineshifts have been studied in the visual solar spectrum for Fe I and Fe II (Dravins et al, 1981; 1986), and in the infrared for Fe I (Nadeau, 1988).

Although significant effects are visible for line-group averages, the laboratory wavelength accuracies are not adequate to test different granulation models using measurements of individual lines only. The noise may be $\simeq 100 \mathrm{~m} / \mathrm{s}$ for stronger Fe I lines, but rapidly gets worse for weaker lines, for high-excitation ones, for Fe II, and for lines in the infrared. Indeed, the lack of sufficiently accurate laboratory data is now the main limiting factor in these studies. Since astronomers do not ordinarily provide such laboratory data, we have to direct our requests to atomic physics groups. The main problem is not the accuracy of measurement, but rather the difficulty of preparing laboratory light sources whose wavelengths are free from systematic effects such as e.g. pressure shifts.

6.2.4. Different Wavelength Shifts in Different Stars. As shown in Fig.7, stars of different temperature and luminosity are predicted to have different amounts of convective lineshift. The vigorous granulation in a $T_{\text {eff }}=6600 \mathrm{~K}$ model of the F5 star Procyon is expected to cause blueshifts in ordinary Fe I lines of $\approx 1000 \mathrm{~m} / \mathrm{s}$, while those in the cooler $\mathrm{T}_{\text {eff }}=5200 \mathrm{~K}$ model amount to only $\simeq 200 \mathrm{~m} / \mathrm{s}$ (Dravins and Nordlund, 1989b). Observed solar values fall in between, typically around $400 \mathrm{~m} / \mathrm{s}$. Understanding such effects is important not only for the study of stellar photospheres per se, but is also required for the accurate determination of stellar radial velocities, in particular in systems with small internal velocity dispersions, such as open galactic clusters.

To separate convective shifts from shifts due to stellar motion, requires one to somehow determine the absolute or relative stellar radial velocity without using the spectral lines. (One also needs to correct for different gravitational redshifts in different stars, but stellar models allow this to be done with good accuracy.)

Differential convective lineshifts should be measurable between stars that share the same space velocity (even if the amount of this velocity is not known). The components of binary stars must share the same system velocity when averaged over their orbits. Thus, visual binaries with not too long periods could be suitable objects to search for spectral-type dependent differences in orbit-averaged apparent radial velocities.

From galactic dynamics arguments, the velocity dispersion of stars in some young galactic clusters is expected to be only a fraction of one $\mathrm{km} / \mathrm{s}$ : less than expected differences in convective lineshift between different spectral types. Thus, if one could identify systematic differences in apparent velocities between different classes of cluster 
members, this would be evidence for different convective lineshifts in different stars.

To determine absolute lineshifts is more challenging since it requires the accurate determination of stellar radial velocities without using any spectral lines nor invoking the Doppler principle. This is possible for the Sun, where the solar motion is well determined from planetary system dynamics (rather than from apparent Doppler shifts of photospheric spectral lines), and consequently solar wavelengths can be corrected for the Sun-Earth motion and compared to laboratory values.

In principle, astrometric measurements could do the same for stars: if one could accurately determine a star's three-dimensional position in space at different times, the difference in position would yield its space velocity. Although current accuracies are insufficient for such measurements of individual stars, the advent of space astrometry promises to make at least some classes of related measurements possible.

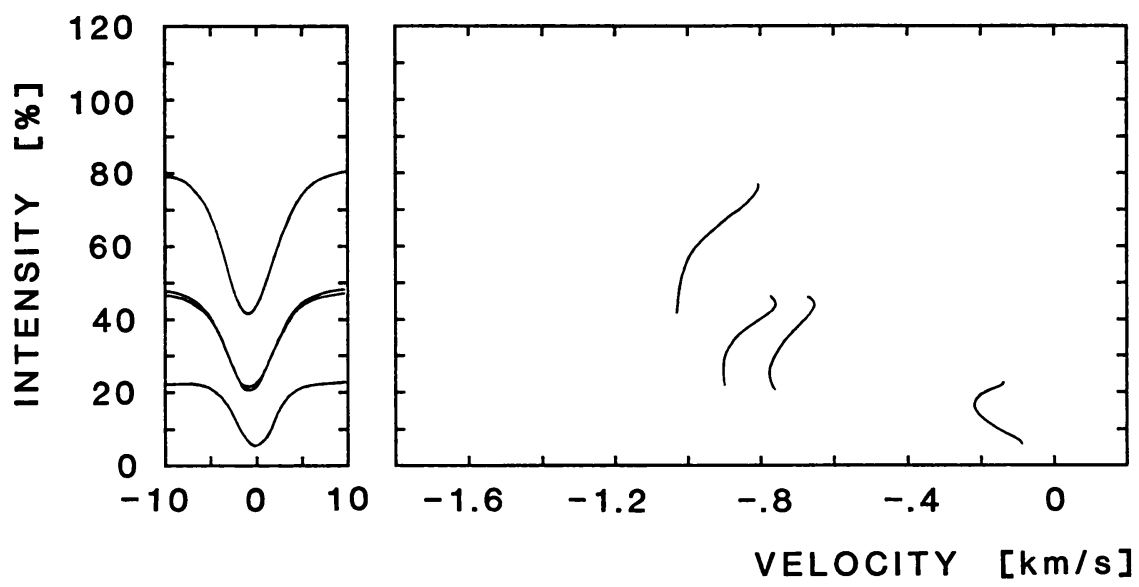

Figure 7. The same spectral line in different stars. Temporally and spatially averaged $\mathrm{Fe} I$ line profiles and bisectors for integrated starlight from four different models are plotted on the same absolute scale. The intensity is normalized to stellar surface area at Procyon disk center, and the wavelength scale is absolute. The oscillator strength for this $\chi=3 \mathrm{eV}$ Fe I line at $\lambda 520 \mathrm{~nm}$ was chosen such that the disk-center absorption depth in the Procyon model is $=60 \%$. From top to bottom, and from left to right, these models represent Procyon (F5 IV-V), $\beta \mathrm{Hyi}$ (G2 IV), $\alpha \mathrm{Cen} A$ (G2 V), and $\alpha \operatorname{Cen} B$ (K1 V). The convective blueshift increases with increasing temperature, and also with increasing luminosity. Observed solar values fall between those of $\alpha \operatorname{Cen} A$ and $\alpha \operatorname{Cen} B$. The corresponding observation of different convective wavelength shifts among different stars remains an observational challenge. A further discussion is in Dravins and Nordlund (1989b).

\subsection{CYCLIC CHANGES OF ASYMMETRIES AND SHIFTS}

A temporal variation in the granulation pattern may change the convective wavelength shifts, and thus mimic a varying stellar radial velocity. To understand such effects could be very important for one very challenging astronomical problem: the search for possible extrasolar planets. 
6.3.1. The Detection of Planets Around Other Stars. Among plausible means of detecting such planets, one of the most promising seems to be the long-term monitoring of the radial velocity of the parent star. If the star is moving in conjunction with an unseen planet, one expects a cyclic change in its velocity. For the Sun-Jupiter system, the amplitude of the solar velocity due to its motion around their common center of gravity is $13 \mathrm{~m} / \mathrm{s}$, with a period of 12 years. There now exist stellar radial velocity instruments with measuring precision sufficient for this task, but there remains the problem of separating cyclic changes due to stellar motion from those due to changes in convective lineshifts.

Solar granulation structure, bisector curvature and amount of lineshift are observed to vary between solar active regions and quiet ones. Consequently, the different area coverage of active regions during different phases of the solar 11-year cycle must lead to changes in the bisector curvature and wavelength shift also in integrated sunlight. Such changes have been studied by Livingston (1983), Deming et al (1987), Jiménez et al. (1988) and Wallace et al (1988).

The exact effect on the apparent radial velocity depends upon precisely how the line is measured, but may correspond to an amplitude of $\approx 30 \mathrm{~m} / \mathrm{s}$ over the solar cycle. Such a magnitude is consistent with observed differences between active and quiet regions, and their different area coverage in different years of the solar cycle. Since many stars possess activity cycles, one should expect qualitatively similar effects in other stars. Clearly, if the Sun, seen from afar, displays an apparent velocity variation of perhaps 30 $\mathrm{m} / \mathrm{s}$ with a period of 11 years, that will not be simple to disentangle from the $13 \mathrm{~m} / \mathrm{s}$ amplitude over 12 years, induced by Jupiter.

To overcome the problem requires a better understanding of stellar granulation properties. Of great importance would be a systematic long-term monitoring of stellar line asymmetries and accurately measured wavelength shifts in different stars. Granulation changes may correlate with active region coverage, and a measure of that is given by the $\mathrm{Ca}$ II $\mathrm{K}$ chromospheric emission intensity. This should also be monitored in order to identify the phase and period of possible stellar activity cycles. True velocity changes must affect all spectral lines, while granulation changes should affect different lines differently. Variations on the shorter timescales of stellar rotation might identify active region patches of significantly modified granulation (Toner and Gray, 1988), while variations over several years might identify the changing area coverage of such features.

\subsection{OBSERVING STRUCTURES ON STELLAR DISKS}

Much of the progress in astronomy is based upon improved spatial resolution. Our understanding of planets, nebulae or galaxies would have been very limited indeed, if we only had observed them as point sources. One of the major aims of stellar granulation studies must be to ultimately observe the fine structure of stellar surfaces and to enable the study also of stars as extended objects. Along with this go related aims, such as understanding the physics of stellar line formation in different atmospheric inhomogeneities. The observational problems are of course caused by the small angular extent of stellar disks - no more than a few tens of milliarcseconds even for the largest stars. Nevertheless, with proper techniques, also the fine structure on stellar disks should become accessible for observation.

6.4.1. Indirect Methods: Deducing Center-to-Limb Line Profile Changes. Line profiles in disk-integrated starlight are built up by contributions from different disk positions. Since both line asymmetry, convective lineshift and continuum brightness depend on the disk position, the integrated line profile incorporates these effects in a complex manner. 
What is needed is a tool to disentangle the different quantities. Such a tool could be available in stellar rotation.

The line profile contributions from different center-to-limb positions, $\cos \theta=\mu$, are not equal for stars of different rotational velocities $V$ sin $i$. Increased rotation changes the Doppler shift at each $\mu$ due to the increased projected velocity, and since the contributions from each $\mu$ have a different line asymmetry, these will be differently distorted by different rotational broadening. For more rapid rotation, when the asymmetric line components originating near the stellar limbs begin to affect the wings of the disk-integrated profile, the bisector patterns change significantly, and the line asymmetries may even become enhanced. This phenomenon was suggested by Gray and Toner (1985; Gray, 1986), and studied in more detail for a simulated rapidly rotating Sun by Smith et al (1987).

Numerical simulations permit the computation of synthetic line profiles for different center-to-limb positions in different stars. From such data, synthetic full-disk line profiles and bisectors are obtained for different rotational velocities $V \sin i$ (Dravins and Nordlund, 1989b). What is still lacking is the observational counterpart: a sequence of line profile, line asymmetry and wavelength shift measurements for groups of stars of the same spectral type, only differing by successively more rapid rotation. Such data could effectively constrain granulation models, and permit the analysis of line profile changes across stellar surfaces.

6.4.2. Direct Methods: Interferometric Imaging of Stellar Surfaces. Although indirect methods might be quite powerful, they do not replace the ultimate need for direct methods to obtain images and spatially resolved spectra across stellar surfaces. The angular (diffraction-limited) resolution of present telescopes in the 4-6 meter class is around 20 milliarcseconds in the visual. Using e.g. speckle interferometry, this allows one to resolve perhaps 10 surface elements on the largest red giants. The forthcoming very large telescopes in the 8-10 m class will significantly improve the situation. Of particular promise is the potential of active optics, which could allow the stable imaging of a stellar disk onto a Fourier transform spectrometer for two-dimensional highresolution spectral observations with good wavelength calibration. It is precisely such data of the center-to-limb changes of stellar line profiles and wavelength shifts that are required to analyze the physics of stellar line formation.

Optical interferometers with baselines on the order of $100 \mathrm{~m}$ and more are now becoming operational, and will offer resolutions around 1 milliarcsecond. That is sufficient for thousands of resolution elements on the disks of red giants, and for resolving nearby main-sequence stars. (The baseline requirements for resolving different classes of stars are discussed by e.g. Dupree et al, 1984.) Since granules on some giant stars might subtend a significant fraction of a stellar diameter, their appearance and spectral features might soon become detectable through e.g. speckle spectroscopy. Solar-type granules, however, have sizes only about one thousandth of the stellar diameter, and their imaging requires baselines a thousand times longer than those required to resolve the stellar disk. To achieve this may require kilometric arrays of space-based optical phase interferometers (or possibly ground-based intensity interferometers), and their feasibility is now under active study by different groups in the world.

\section{ACKNOWLEDGEMENT}

This work is supported by the Swedish Natural Science Research Council 


\section{References}

Antia, H.M., Chitre, S.M., Narashima, D.: 1984, Astrophys.J. 282, 574

Atroshchenko, I.N., Gadun, A.S., Kostik, R.I.: 1989, in R.F.Rutten, G.Severino, eds. Solar and Stellar Granulation, Kluwer, p. 521

Bowler, K.C., Bruce, A.D., Kenway. R.D., Pawley, G.S., Wallace, D.J.: 1987, Physics Today 40, No. 10, p. 40

Bray, R.J., Loughhead, R.E.: 1978, Astrophys.J. 224, 276

Deming, D., Espenak, F., Jennings, D.E., Brault, J.W., Wagner, J.: 1987, Astrophys. J. 316, 771

Dravins, D.: 1981, Astron. Astrophys. 98, 367

Dravins, D.: 1982, Ann. Rev. Astron. Astrophys. 20, 61

Dravins, D.: 1987a, Astron. Astrophys. 172, 200

Dravins, D.: 1987b, Astron. Astrophys. 172, 211

Dravins, D.: 1989, in R.J.Rutten, G.Severino, eds. Solar and Stellar Granulation, Kluwer, p. 493

Dravins, D., Larsson, B., Nordlund, Å.: 1986, Astron. Astrophys. 158, 83

Dravins, D., Lindegren, L., Nordlund, Å.: 1981, Astron. Astrophys. 96, 345

Dravins, D., Nordlund, Å.: 1989a, Astron. Astrophys., in press

Dravins, D., Nordlund, $\AA .:$ 1989b, Astron. Astrophys., in press

Dupree, A.K., Baliunas, S.L., Guinan, E.F.: 1984, Bull.AAS 16, 797

Gigas, D.: 1989, in R.F.Rutten, G.Severino, eds. Solar and Stellar Granulation, Kluwer, p. 533

Gray, D.F.: 1986, Publ.Astron. Soc. Pacific 98, 319

Gray, D.F.: 1988, Lectures on Spectral-Line Analysis: F, G, and K Stars, The Publisher, Arva (Ontario)

Gray, D.F., Nagel, T.: 1989, preprint

Gray, D.F., Toner, C.G.: 1985, Publ. Astron. Soc. Pacific 97,543

Gray, D.F., Toner, C.G.: 1986, Publ.Astron. Soc. Pacific 98, 499

Gray, D.F., Toner, C.G.: 1985, Publ. Astron. Soc. Pacific 97, 543

Jiménez, A., Palle, P.L., Régulo, C., Roca Cortés, T., Elsworth, Y.P., Isaak, G.R., Jefferies, S.M., McLeod, C.P., New, R., van der Raay, H.B.: 1988, in J.Christensen-Dalsgaard, S. Frandsen, eds. Advances in Helio- and Asteroseismology, Reidel, IAU symp 123, p. 215

Livingston, W.C.: 1983, in J.O.Stenflo, ed. Solar and Stellar Magnetic Fields: Origins and Coronal Effects, Reidel, IAU symp. 102, p. 149

Livingston, W., Huang, Y.R.: 1986, in M.S.Giampapa, ed. The SHIRSHOG Workshop, National Solar Observatory, Tucson, p. 1

Nadeau, D.: 1988, Astrophys.J. 325, 480

Nadeau, D., Maillard, J.P.: 1988, Astrophys.J. 327, 321

Nelson, G.D.: 1980, Astrophys.J. 238, 659

Nordlund, A.: 1982, Astron. Astrophys. 107, 1

Nordlund, Å.: 1985, Solar Phys. 100, 209

Nordlund, A., Dravins, D.: 1989, Astron. Astrophys., in press

Schwarzschild, M.: 1975, Astrophys.J. 195, 137

Smith, M.A., Huang, Y.R., Livingston, W.: 1987, Publ.Astron. Soc. Pacific 99, 297

Smith, M.A., Karp, A.H.: 1978, Astrophys. J. 219, 522

Smith, M.A., Karp, A.H.: 1979, Astrophys.J. 230, 156

Toner, C.G., Gray, D.F.: 1988, Astrophys. J., 334, 1008

Wallace, L., Huang, Y.R., Livingston, W.: 1988, Astrophys. J. 327, 399

Wayte, R.C., Ring.J.: 1977, Monthly Notices Roy. Astron. Soc. 181, 131

Winkler, K.H.A., Chalmers, J.W., Hodson, S.W., Woodward, P.R., Zabusky, N.J.: 1987, Physics Today 40, No.10, p. 28 